\title{
Intersectoral Cost of Treating Pulmonary Non-Tuberculosis Mycobacterial Disease (NTM-PD) in Germany-A Change of Perspective in Disease Management
}

\author{
Roland Diel ${ }^{1,2,3, *}$ and Pontus Mertsch ${ }^{4}$ \\ 1 Institute for Epidemiology, University Medical Hospital Schleswig-Holstein, Niemannsweg 11, \\ 24015 Kiel, Germany \\ 2 LungClinc Grosshansdorf, Airway Research Center North (ARCN), Member of the German Center for Lung \\ Research (DZL), 22927 Großhansdorf, Germany \\ 3 Institution for Statutory Accident Insurance and Prevention in the Health and Welfare Services (BGW), \\ 22089 Hamburg, Germany \\ 4 Department of Internal Medicine V, Ludwig-Maximilians-University of Munich, Comprehensive \\ Pneumology Center (CPC-M), Member of the German Center for Lung Research (DZL), \\ 80336 Munich, Germany; Pontus.Mertsch@med.uni-muenchen.de \\ * Correspondence: roland.diel@epi.uni-kiel.de; Tel.: +49-(0)-1724578525; Fax: +49-(0)-4315973730
}

Received: 5 September 2019; Accepted: 5 October 2019; Published: 9 October 2019

\begin{abstract}
Background: In line with its increasing prevalence, pulmonary Mycobacterium avium complex (MAC) disease (MAC-PD) gives rise to substantial healthcare costs. However, there is only limited information on the costs of intersectoral reimbursement. Objectives: Inpatient and outpatient costs for diagnosing and treating pulmonary MAC-PD in Germany in accordance with standard international guidelines were calculated and their potential effects on MAC disease management in Germany were determined. Methods: Hospitalization costs were calculated by using the German diagnosis related group (G-DRG) browser, with and without inclusion of the diseases most often associated with M. avium. Separated by drug macrolide susceptibility and severity of MAC-PD, the direct medical costs of suitable therapies in the outpatient setting were determined by Monte-Carlo simulation, including all conceivable options. Results: According to our simulation, the weighted mean cost of outpatient treatment over 14 or 18 months, in either case followed by a post-treatment monitoring over 12 months, amounts to $€ 8675.22$ (95\% confidence interval [CI] $€ 8616.17$ to $€ 8734.27$ ). Of that amount, the revenue for outpatient doctors' services, dependent on treatment duration, is low, ranging between $€ 894.79$ (10.3\%) and $€ 979.42$ (11.3\%), accordingly. Mean drug costs for MAC-PD patients amount to $€ 6130.25$ [95\% CI $€ 6073.52$ to $€ 6186.98$ ], i.e., more than two third (70.7\%) of the total outpatient costs. In contrast, the non-surgical reimbursement for a hospital stay of up to 14 days is $€ 3321.64$. Hospital reimbursement does not increase in cases of complications (a higher number and/or challenging type of associated diseases), but it is fully paid even in cases that require as few as 2 days of hospitalization. Conclusion: The imbalance between well-rewarded hospital care and the low reimbursement for long-term treatment of MAC-PD outpatients may induce inappropriate disease management. In order to arrive at properly integrated care of MAC-PD patients in Germany, measures such as better incentives for physicians in the outpatient setting and a targeted use of resources in hospitals are required. Reimbursed, periodic case conferences between outpatient physicians and experts in hospitals as well as preventive short-term checks of MAC-PD patients in specialty clinics may promote cross-sector cooperation and improve overall treatment quality. Nationwide pilot studies are required to gain evidence on the effectiveness of the new approach.
\end{abstract}


Keywords: cost analysis; Monte Carlo simulation; non-tuberculosis mycobacteria; Mycobacterium avium; integrated care; healthcare management

\section{Introduction}

Pulmonary disease caused by non-tuberculous mycobacteria (NTM-PD) is a growing, worldwide public health challenge [1], whereby species of Mycobacterium avium complex (MAC), i.e., M. avium, $\mathrm{M}$. intracellulare, and M. chimaera, are the most common pulmonary NTM pathogens in almost all regions of the world [2,3].

The annual increase in NTM-PD prevalence in recent years has been shown to be more than $8 \%$ in the United States [4], and recently, Ringshausen et al. observed a significant increase of $5.9 \%$ per year in overall age-adjusted NTM-PD-associated hospitalizations in Germany [5]. In South Korea, the age-adjusted NTM prevalence is estimated to be 33.3 per 100,000 population in 2016 [6].

NTM-PD-associated morbidity is characterized by creeping destruction of the patient's lungs, and places significant economic burdens on the health care system. Based on administrative data of the German statutory health insurance community (SHI), the mean direct expenditure per NTM-PD patient in the 39 months following first diagnosis was $€ 39,559.60$, nearly four times that for a matched control [7]. During this observational period, a clearly higher mortality rate occurred in patients with NTM-PD $(22.4 \%)$ as compared to patients of the control group $(6 \%)(p<0.001)$.

Hospital costs are three times higher in the NTM-PD group than in the matched non-NTM-PD group. However, most patients (52\%) are initially diagnosed and treated as outpatients (unpublished original data for [7]) although in Germany there is generally no waiting time for access to pulmonological hospital departments or lung clinics.

Of note, during the second year of treatment, $31.1 \%$ of the surviving NTM patients had to be either hospitalized for the first time or re-hospitalized [7], suggesting that the choice of medication and/or treatment adherence in the NTM-PD group may have been, at least in part, ineffective. In effect, hospitalization proves to be the main cost driver in resolving NTM-PD cases.

According to the available SHI data, only $54.4 \%$ of patients began treatment within three months of a new NTM-PD diagnosis, and median time between (discharge) diagnosis and therapy initiation was 32 days (interquartile range [IQR] 260 days), revealing some uncertainty about which basic regimens should be chosen [7]. This is of high importance, as a recently published study analyzing data from a large US managed care provider claims [8]. There, guideline base treatment (GBT) was associated with a significantly lower all-cause hospitalization risk versus non-GBT (odds ratio 0.53 ) and adjusted total health-care expenditure in year 2 with GBT was clearly lower than that with non-GBT, the difference being $\$ 7933$.

The high economic burden of pulmonary NTM disease in Germany raises the question as to how better disease management may contribute to improved clinical outcomes. An essential structural feature of the German health care system is its strict separation into two different sectors, the outpatient and the hospital sector. Although both healthcare provider types are paid by the SHI, reimbursement to each sectors follows the sector's own system and accounting procedures, namely the Statutory Health Insurance Scheme (Einheitlicher Bewertungsmaßstab, EBM) [9] for ambulatory services and the German diagnosis related complex system (G-DRG) for hospital stays, updated each year by the InEK (Institute for the Hospital Remuneration System [10]). A special form applicable to cases of MAC-PD, the so-called ambulatory specialized care ("ambulante spezialärztliche Versorgung, ASV"), is defined in paragraph 116 b SGB V [Volume V of the Social Insurance Code (Fünftes Buch Sozialgesetzbuch—SGB V)] and was implemented in 2012 for continuing tuberculosis (TB) or NTM-PD treatment. It may be used in both sectors, but is applicable only under the EBM system [11].

To date, the only data available for the costs in Germany of treating NTM patients, Diel's burden of illness study, [7] is confounded at least in part by the inclusion there of patients with concomitant 
diseases in the NTM group as well as in the matched non-NTM group. A systematic analysis of the costs occurring exclusively for diagnosing, monitoring, and treating of NTM-PD in the two German sectors, including the inherent problems of each, has not, to date, been published. Our analysis also aims to clarify options for overcoming structural weaknesses in the treatment of NTM-PD, based on the example of Mycobacterium avium complex, the most commonly isolated pathogen in NTM-PD worldwide.

\section{Material and Methods}

This analysis considers the charging of direct medical costs under the current status quo for diagnosis and therapy of pulmonary MAC disease as accepted and borne by the SHI.

Our calculations of the direct costs of MAC-PD outpatients are therefore based on the drug costs occurring under different treatment combinations and on the rates established by the Statutory Health Insurance Scheme (EBM) [9] for outpatient services. Costs of inpatients were assessed using data provided by the InEK for the G-DRG system [10].

According to international recommendations, four different drug combinations for a standard treatment of macrolid-susceptible MAC-PD cases, two for macrolid-resistant MAC-PD and four for severe MAC-PD, have to be taken into consideration (for details see Supplementary material, File S1). The drug costs of each treatment option were determined and the assumed frequency of these options (probability weight) was applied. To calculate the mean value and the $95 \%$ confidence interval (CI) of the drug costs of all treatment options, a "random walk" through the 10 different options was made by Monte Carlo simulation [12], with 10,000 simulation runs performed.

Subsequently, the drug costs of the respective treatment regimens were each added to the separately calculated costs of treating and monitoring outpatients, either for 26 or 30 months (see below for details), arriving at 20 different options. Again, the probability weight of each option was applied, whereby-due to uncertainty on how often a shorter or longer treatment is required in a pulmonary MAC patient- the two treatment durations were given the same probability (see Table 2). To calculate the mean value and the $95 \%$ confidence interval (CI) of the total outpatient costs, a random walk was made through the 20 different options by a second Monte Carlo simulation, again with 10,000 simulation runs performed.

\subsection{Basic Assumptions for Calculating Outpatients Drug Costs}

As a first step in our modeling, we assume that general practitioners refer afflicted patients either directly to a hospital, or to a lung specialist in a private practice. Accordingly, the patient will undergo diagnostic procedures and therapy, either as a primary inpatient or as a primary outpatient throughout the treatment and port-treatment monitoring period. Drug costs for outpatients are determined by selecting the least expensive alternatives from the pharmacy retail price catalogue (Apothekenverkaufspreis, AVP), which is part of the Red List (Rote Liste ${ }^{\circledR}$ ) 2019, Germany's register of pharmaceutical drugs [13]. Discount agreements for drug costs, an option open to single health insurance organizations under the German system, are not considered in our calculations. The drugs and the abbreviations used in the model are listed in Table 1. Daily therapy costs were calculated by dividing pack quantity by the drug's recommended daily intake as stated in the dosing instructions.

Unfortunately, patterns of resistance in MAC-PD have not yet been systematically studied, and data in the literature are sparse. Generally, a selection of three of six drugs taken daily is recommended for the entire 14 or 18 months of treatment. The choices are a macrolide (clarithromycin (CLAM) or azithromycin $(A Z M)$ ), ethambutol (E), and rifampicin (R) or rifabutin (RBT) in macrolide-susceptible MAC-PD patients, and in macrolide-resistant cases, clofazimin (CLO) or a fluorquinolone, e.g., moxifloxacin (MOX), instead of a macrolide. Amikacin (AMK) should be administered intravenously for at least eight weeks in macrolide-resistant and/or severe MAC-PD cases (for details see Supplement). As sputum conversions generally occur only after 2-6 months of treatment, and since 12 months of further 
treatment should follow conversion, the typical MAC-PD patient will receive treatment for 14 to 18 months.

Table 1. List of medical drugs for the treatment of pulmonary disease caused by non-tuberculous mycobacteria (MAC-PD) available in Germany.

\begin{tabular}{llll}
\hline Name & Abbreviation & Form/Price & Daily Dose \\
\hline Amikacin FRESENIUS & AMK & 10 Infusion bottles - & $2 \times 1: € 71.40$ \\
Clarithromycin-ratiopharm 500 mg & CLAM & Fl. $500 \mathrm{mg} / 100 \mathrm{~mL}: € 357.0$ & $2 \times 1: € 1.95$ \\
Zithromax 250 mg & AZM & 6 film-coated tablets: $€ 19.52$ & $1 \times 1: € 3.46$ \\
Clofazimin (LAMPREN) $100 \mathrm{mg}$ & CLO & $€ 20.77$ & $1 \times 1: € 2.0$ \\
Ethambutol (EMB-Fatol) $400 \mathrm{mg}$ & E & 100 tablets: $€ 199.92$ & $3 \times 1: € 1.25$ \\
Moxifloxacin HEXAL 400 mg & MOX & 100 film-coated tablets: $€ 41.75$ & $1 \times 1: € 3.97$ \\
Eremfat 600 mg & R & 10 film-coated tablets: $€ 39.73$ & $1 \times 1: € 2.71$ \\
Rifabutin MYCOBUTIN PFIZER 150 mg & RBT & 271.46 film-coated tablets: & $2 \times 1: € 10.87$ \\
\hline
\end{tabular}

As the actual composition of drug regimens administered to MAC-PD patients in Germany is not known, weighting of possible treatment schedules was required in our modeling. From the literature, we found that Medical records of 634 human immuno-deficiency virus [HIV]-negative patients with pulmonary MAC disease in Japan showed fibrocavitary disease in $16.6 \%$ of patients [14]. Griffith et al. [15] reported a similar rate of macrolide resistance (15.92\%) in 565 patients with MAC lung disease. Thus, we assumed that generally $84 \%$ of MAC patients could be treated without AMK. For the remaining $14 \%$, the probabilities of the possible regimens were considered to be equally distributed (see Table 2).

Table 2. Total costs of diagnosing, monitoring, and treating pulmonary MAC disease in German outpatients.

\begin{tabular}{|c|c|c|c|c|c|c|c|}
\hline $\begin{array}{c}\text { Medication } \\
\text { (Possible Drug } \\
\text { Combinations) }\end{array}$ & $\begin{array}{l}\text { Drug } \\
\text { Costs/Day } \\
\text { in } €\end{array}$ & $\begin{array}{l}\text { Drug } \\
\text { Costs } 14 \\
\text { Months } \\
\text { in } €\end{array}$ & $\begin{array}{l}\text { Drug Costs } 18 \\
\text { Months in } €\end{array}$ & $\begin{array}{c}\text { Costs of } \\
\text { Diagnostics/Monitoring } \\
14 \text { Months in } €\end{array}$ & $\begin{array}{c}\text { Costs of } \\
\text { Diagnostics/ } \\
\text { Monitoring 18 } \\
\text { Months in } €\end{array}$ & $\begin{array}{c}\text { Total } \\
\text { Outpatient } \\
\text { Cost 14 Months } \\
\text { in } €\end{array}$ & $\begin{array}{c}\text { Total } \\
\text { Outpatient } \\
\text { Cost } 18 \text { Months } \\
\text { in } €\end{array}$ \\
\hline \multicolumn{8}{|c|}{ Macrolid-susceptible MAC-PD } \\
\hline R-E-CLAM & 5.91 & 2482.2 & 3191.4 & 2162.47 & 2895.75 & $4644.67(0.84 / 8)$ & $6087.15(0.84 / 8)$ \\
\hline RBT-E-CLAM & 14.08 & 5913.6 & 7603.2 & 2162.47 & 2895.75 & $8076.07(0.84 / 8)$ & $\begin{array}{c}10,498.95 \\
(0.84 / 8)\end{array}$ \\
\hline R-E-AZM & 7.42 & 3116.4 & 4006.8 & 2162.47 & 2895.75 & $5287.87(0.84 / 8)$ & $6902.55(0.84 / 8)$ \\
\hline RBT-E-AZM & 19.54 & 8206.8 & $10,551.6$ & 2162.47 & 2895.75 & $\begin{array}{c}10,369.27 \\
(0.84 / 8)\end{array}$ & $\begin{array}{c}13,447.35 \\
(0.84 / 8)\end{array}$ \\
\hline \multicolumn{8}{|c|}{ Macrolid-resistant MAC-PD } \\
\hline $\begin{array}{l}\text { R-E-CLO-AMK } \\
\text { (AMK } 60 \text { days) }\end{array}$ & $5.96(4284)$ & 6787.2 & 7502.4 & 2263.3 & 2996.58 & $9050.5(0.16 / 12)$ & $\begin{array}{l}10,498.98 \\
(0.16 / 12)\end{array}$ \\
\hline $\begin{array}{l}\text { R-E-MOX-AMK } \\
\text { (AMK } 60 \text { days) }\end{array}$ & $7.93(4284)$ & 7614.6 & 8556.2 & 2263.3 & 2996.58 & $9877.9(0.16 / 12)$ & $\begin{array}{l}11,552.78 \\
(0.16 / 12)\end{array}$ \\
\hline \multicolumn{8}{|c|}{ Severe NTM } \\
\hline $\begin{array}{l}\text { R-E-CLAM-AMX } \\
\text { (AMK } 60 \text { days) }\end{array}$ & $5,91(4284)$ & 6766.2 & 7475.4 & 2263.3 & 2996.58 & $9029.5(0.16 / 12)$ & $\begin{array}{l}10,471.98 \\
(0.16 / 12)\end{array}$ \\
\hline $\begin{array}{l}\text { RBT-E-CLAM-AMX } \\
\text { (AMK } 60 \text { days) }\end{array}$ & $\begin{array}{l}14.08 \\
(4284) \\
\end{array}$ & $10,197.6$ & $11,887.2$ & 2263.3 & 2996.58 & $\begin{array}{l}12,460.9 \\
(0.16 / 12) \\
\end{array}$ & $\begin{array}{l}14,883.78 \\
(0.16 / 12) \\
\end{array}$ \\
\hline $\begin{array}{l}\text { R-E-ATZ-AMX } \\
\text { (AMK } 60 \text { days) }\end{array}$ & $7.42(4284)$ & 7400.4 & 8290.8 & 2263.3 & 2996.58 & $9663.7(0.16 / 12)$ & $\begin{array}{l}11,287.38 \\
(0.16 / 12)\end{array}$ \\
\hline $\begin{array}{l}\text { RBT-E-ATM-AMX } \\
\text { (AMK } 60 \text { days) }\end{array}$ & $\begin{array}{c}19.54 \\
(4284)\end{array}$ & $12,490.8$ & $12,840.2$ & 2263.3 & 2996.58 & $\begin{array}{l}14,754.1 \\
(0.16 / 12)\end{array}$ & $\begin{array}{l}15,806.78 \\
(0.16 / 12)\end{array}$ \\
\hline
\end{tabular}

\subsection{Assumed Procedures in the Diagnosis and Monitoring of MAC-PD}

\section{Microbiology:}

Initially, to verify that the strain represent disease rather than colonization, three separate expectorated sputum samples were collected on separate days for microscopy and sent for culturing. 
The procedure was repeated under treatment every four weeks until negative results were obtained, i.e., at least five times for a presumed conversion within eight weeks or nine times for a presumed conversion within 6 months. The first positive culture in the initial phase is subjected to resistance testing.

NTM-PD patients must be strictly monitored: following culture conversion, sputum smear microscopy and culture examination are performed at least every 3 months (BTS) throughout the 12-month continued drug therapy and subsequently every 3 months for 12 months, i.e., eight times. In total, the number of microscopies and cultures before, during and after treatment, amounts to 13 or-if culture conversion cannot be achieved within 6 months—-to 17 repetitions.

2. Blood work:

A broad blood profile was necessary before therapy started. Kidney retention values (creatinine, urea) and liver values (glutamic oxaloacetic transaminase [GOT], glutamate pyruvate transaminase [GPT], bilirubin, gamma-glutamyltransferase [GGT]) are key. GGT is required as an alcohol abuse parameter and also allows as an indicator for Rifampin-induced cholangitis. It is also advisable to establish a serological profile for hepatitis (hepatitis B virus surface antigen [HBs-Ag], antibodies to hepatitis B core [Anti-HBc]) and HIV. Liver values must be controlled 2-4 weeks after starting therapy, and every 4 weeks thereafter. The renal retention parameters are checked every month through to the end of the therapy, together with the liver values.

Serum electrolytes and CRP are checked every 2 weeks for 2 months, then monthly in all patients. If intravenous AMK has been prescribed as an additional drug, the serum level of AMK should be controlled at the end of the first week, and again 2 weeks and 6 weeks after starting the therapy (given normal kidney function).

3. Ophthalmic examination: under EMB, before therapy starts and usually every 4 weeks, i.e., 14 to 18 times in total. If AMK has been prescribed, audiometry was performed in the initial phase and then every 4 weeks during therapy, altogether three in total.

4. X-rays are recommended in the initial phase, after 4 weeks (to control if the NTM is reacting to the therapy) and after 8 weeks (end of the initial therapy: success assessment). After 8 weeks, $X$-ray checkups in the 4 th and 6th month are sufficient, followed by check-ups every 3 months and 6,12 , and 24 months after the end of treatment, amounting to 11 in total. The number of X-rays increases to 15 if culture conversion cannot be achieved with 6 months of treatment.

5. Computer tomography will be used as a diagnostic supplement prior to therapy and then every 6 months, i.e., three times in total.

6. ECG monitoring has to be performed when using drugs that may prolong corrected QT interval [QTc](CLAM or AZM). ECG is recommended at $0,2,12$, and 24 weeks.

\section{Results}

\subsection{Outpatient Costs}

The costs of all reasonable treatment regimens under consideration, separated by drug costs for a treatment of 14 or 18 months, and the remaining costs are depicted in detail in Table 2. According to the results of the first order Monte Carlo simulation that takes account of treatment variability, the weighted mean drug costs were $€ 6130.25$ [95\% CI $€ 6073.52$ to $€ 6186.98]$. The remaining costs of diagnosing, treating, and monitoring pulmonary MAC disease (under the standard course of therapy) are $€ 2162.47$ for a 14-month course of treatment and $€ 2895.75$ for 18 months. The addition of intravenous AMK in severe or macrolide-resistant cases, increases the costs to $€ 2263.3$ for a 14 month and $€ 2996.58$ for a 18 month course of treatment (see Table 3). The weighted mean of outpatient treatment cost over 14 or 18 months, followed by a post-treatment monitoring over 12 months, as calculated by the second Monte Carlo simulation, is $€ 8675.22$ (95\% CI €8616.17 to €8734.27). 
Table 3. Weighted mean of outpatient costs *

\begin{tabular}{cc}
\hline Statistic & Value \\
\hline Mean & $€ 8675.22$ \\
Standard deviation & $\pm € 3012.67$ \\
Minimum & $€ 4644.67$ \\
Median & $€ 8076.07$ \\
95\% Confidence Interval & $€ 8616.17$ to $€ 8734.27$ \\
Maximum & $€ 15,806.78$ \\
Sum $(\mathrm{n} \times$ Mean) & $€ 86,752.202 .82$ \\
Size (n) & 10,000 \\
Variance & $€ 9076,177.69$ \\
Variance/Size & $€ 907.62$ \\
SQRT[Variance/Size] & $€ 30.13$ \\
\hline * Cost weight calculated by using Monte Carlo simulation.
\end{tabular}

Of that amount, the revenue for outpatient doctors' services themselves only plays a minor role; in Germany, the outpatient doctors are paid per quarter. Thus, for the shorter treatment duration the revenue refers to nine quarters, or 27 months, and for the longer treatment duration to 10 quarters, or 30 months. According to the figures presented in Table 4, the revenue for the GP is $€ 517.14$ for the shorter treatment of 14 months plus the 12-month monitoring period and $€ 574.60$ for the longer treatment duration of 18 months. The pulmonologists receive $€ 377.65$ and $€ 404.82$, respectively. Dividing these revenues by 26 or 30 months the (rounded) revenue per month for the GP is on average $€ 19.89$ ( $€ 517.14 / 26)$ and $€ 14.53$ ( $€ 377.65 / 26)$ for the pulmonologist or $€ 19.15$ ( $5574.6 / 30)$ and $€ 13.49$ (€404.82/30). That means that-dependent on treatment duration—the revenue for outpatient doctors (adding the revenues for the GP and the pulmonologist together) per month is on average $€ 34.42$ or $€ 32.65$.

Summing up the revenue for the GP and the pulmonologist to one figure, according to Table 4, the percentage share of the costs for outpatient doctors' services of the weighted mean of total outpatient costs is $10.3 \%$ ( $€ 894.79 / € 8675.22)(10.3 \%)$ for a total of 26 months or $11.3 \%$ (€979.42/€8675.22), for a total of 30 months.

\subsection{Specialized Ambulatory Care (ASV)}

Since 2012, ambulatory specialized care for TB and NTM can be conducted in Germany under strict conditions in accordance with section 116b SGB V in both the outpatient setting and hospitals. However, contracted physicians and hospitals intending to implement such an ASV must provide their services at the same (low) rates as with purely outpatient remuneration (EBM).

Furthermore, the prerequisites for maintaining an ASV are very strict; per year, at least 20 patients with TB or NTM have to be treated in an interdisciplinary team with a pulmonologist as director and under settled doctor's conditions. Beyond the outpatient-based remuneration, full-time equivalents for doctors or nursing in the hospital that establishes such a service will not be paid. Thus, a hospital can cover the actual costs of such ambulatory care only with a pre-existing high organizational overhead. On the other hand, the number of new TB or NTM cases a single outpatient doctor will see in a given year is generally well below the 20 or more required for an ASV.

Accordingly, for all participants, whether hospital or outpatient doctors, the procedure of furnishing the continually requested proof that the conditions of participation can be fulfilled is burdensome. Therefore, it is no surprise that the willingness to engage in ambulatory specialized care is very low; to date, only 34 ASV for treating mycobacterial diseases currently exist in Germany, of those 27 in hospitals [9]. 
Table 4. German statutory health insurance community (SHI) costs of diagnosing and monitoring pulmonary NTM in the outpatient setting.

\begin{tabular}{|c|c|c|c|c|}
\hline Medical Services & No. of Points & Individual Payment $(€)$ & Frequency & Payment (€) \\
\hline \multicolumn{5}{|c|}{ General practitioner } \\
\hline $\begin{array}{l}\text { Flat rate coverage (irrespective of number of visits by patients) per } \\
\text { quarter; (EBM Gebührenordnungsposition (GOP) } 03000\end{array}$ & 157 & 16.99 & 9 [quarters] (10) & $152.91(169.9)$ \\
\hline $\begin{array}{c}\text { GOP 03220, additional fee on GOP } 03000 \text { (due to NTM as chronic } \\
\text { illness, "Chronikerzuschlag") }\end{array}$ & 130 & 14.07 & $9(10)$ & $126.63(140.7)$ \\
\hline GOP 03222 , additional fee on GOP 03220 & 10 & 1.08 & $9(10)$ & $9.72(10.8)$ \\
\hline Detailed conversation (GOP04230) & 90 & 9.74 & $9(10)$ & $87.66(97.40)$ \\
\hline Retainer fee (general practitioner), once per quarter (GOP 03040) & 144 & 15.58 & $9(10)$ & $140.22(155.80)$ \\
\hline \multicolumn{5}{|c|}{ Pneumological diagnostics (NTM) } \\
\hline Pneumological consultation; 60 years of age and above (GOP 13642) & 210 & 22.73 & $9(10)$ & $204.57(227.30)$ \\
\hline Surcharge (pneumologist), once per quarter (GOP 13644) & 41 & 4.44 & $9(10)$ & $39.96(44.40)$ \\
\hline Bronchoscopy (GOP 13662) & 988 & 106.93 & 1 & 106.93 \\
\hline Pneumologist BAL (GOP 13663), additional fee on GOP 13662 & 242 & 26.19 & 1 & 26.19 \\
\hline \multicolumn{5}{|c|}{ Methods } \\
\hline ECG (EBM 27320) & \multicolumn{4}{|c|}{ Cannot be charged separately } \\
\hline $\begin{array}{c}\text { Audiometry (OPS 09320), ENT code can also be applied (before } \\
\text { starting therapy with macrolides) }\end{array}$ & 147 & 15.91 & $\begin{array}{l}3 \text { (initially and then once } \\
\text { per month) }\end{array}$ & 47.73 \\
\hline X-ray (EBM 34241; a consultation cannot be charged) & 152 & 16.45 & $11(15)$ & $180.95(246.75)$ \\
\hline Ophthalmologic consultation (EBM 06212) & 150 & 16.23 & 14 [once per month] (18) & $227.22(292.14)$ \\
\hline Computerized tomography (EBM 34330) & 660 & 71.43 & $\begin{array}{c}3 \text { [initially and once per } \\
\text { half year] (4) }\end{array}$ & $214.29(285.72)$ \\
\hline Surcharge contrast agent (EBM 34345) & 228 & 24.68 & $3(4)$ & $74.04(98.72)$ \\
\hline \multicolumn{5}{|c|}{ Microbiology } \\
\hline Microscopy test for mycobacteria (EBM 32176) & - & 5.20 & $13(17)$ & $67.6(88.4)$ \\
\hline NAAT (EBM 32825) & - & 61.40 & 1 & 61.40 \\
\hline Culture test for mycobacteria (EBM 32747) per material & - & 34.90 & $13(17)$ & $453.7(593.3)$ \\
\hline Differentiation of mycobacteria (EBM 32764) if positive & - & 28.40 & 1 & 28.40 \\
\hline $\begin{array}{l}\text { Resistance definition (EBM } 32770 \text { ) per mycobacteria type (repeating } \\
\text { of susceptibility testing only required if patient recultures MAC after } \\
\text { culture conversion) }\end{array}$ & - & 39.50 & 1 & 39.50 \\
\hline
\end{tabular}


Table 4. Cont.

\begin{tabular}{|c|c|c|c|c|}
\hline Medical Services & No. of Points & Individual Payment (€) & Frequency & Payment (€) \\
\hline \multicolumn{5}{|c|}{ Laboratory investigation } \\
\hline Anti-HBc (EBM 32614) & - & 5.90 & 1 & 5.90 \\
\hline HBs-Ag (EBM 32781) & - & 5.50 & 1 & 5.50 \\
\hline Chlorid (EBM 32084) & - & 0.25 & $\begin{array}{c}16 \text { [every } 2 \text { weeks for } \\
2 \text { months, then once per } \\
\text { month] (20) }\end{array}$ & $4(5)$ \\
\hline Natrium (EBM 32083) & - & 0.25 & $16(20)$ & $4(5)$ \\
\hline Potassium (EBM 32081) & - & 0.25 & $16(20)$ & $4(5)$ \\
\hline Calcium (EBM 32082) & - & 0.25 & $16(20)$ & $4(5)$ \\
\hline Creatinine (Jaffe method) (EBM 32066) & - & 0.25 & $16(20)$ & $4(5)$ \\
\hline Urea (EBM 32065) & - & 0.25 & $16(20)$ & $4(5)$ \\
\hline Blood count (EBM 32122) & - & 1.10 & $16(20)$ & $17.6(22.20)$ \\
\hline Bilirubin total (EBM 32058) & - & 0.25 & $16(20)$ & $4(5)$ \\
\hline Gamma-glutamyl transferase (EBM 32071) & - & 0.25 & $16(20)$ & $4(5)$ \\
\hline Glutamate-oxaloacetate transaminase (EBM 32069) & - & 0.25 & $16(20)$ & $4(5)$ \\
\hline Glutamate-pyruvate transaminase (EBM 32070) & - & 0.25 & $16(20)$ & $4(5)$ \\
\hline CRP (EBM 32460) & - & 4.90 & $16(20)$ & $78.4(98)$ \\
\hline Amikacin serum levels (EBM 32341) & - & 17.70 & 3 & 53.10 \\
\hline
\end{tabular}

Anti-HBc = Hepatitis B core antibody; BAL = bronchoalveolar lavage; ENT = Ear Nose Throat; HBs-Ag = surface antigen of the hepatitis B virus; NAAT = Nucleic Acid Amplification Test. 


\subsection{Hospital Reimbursement}

As far as in-patients are concerned, since January 1st, 2004, hospital costs are based on the uniform German G-DRG system, which allocates each case to a diagnosis-related group. Under that system, reimbursement of hospital services is no longer based on fixed daily rates for the period of stay, but focuses on the type and severity of the diseases and the coding of the medical operations and procedures according to the German Procedure Classification (Operation and Procedure Code, OPS), e.g., diagnostic bronchoscopy (OPS 1-690.0) or thoracic computer tomography (OPS 3-222), performed for the respective NTM patient during the hospital stay. Diagnosis of the respective diseases of the inpatients, with the associated procedures performed, must be coded by the responsible physicians using the respective German (G)-ICD 10 list and entered in an officially approved grouper (GetDRG [16]). A national base rate for the hospitals that is updated annually by the InEK is then multiplied by the specific cost weight of a disease that will be calculated according to an internal algorithm of the IneK, resulting in the effective reimbursement for the hospital. Usually, a hospital stay of NTM-PD patients in Germany is 14 days [10], of which the admission and discharge day are each credited only as half and pulmonary NTM disease (ICD-10 A31.x, infections by other mycobacteria) will be grouped into the DRG E76C (TB without severe complications, hospital stay up to 14 days).

Of note, with the exception of cystic fibrosis (CF), inserting all reasonable combinations of NTM as main diagnosis in the grouper together with well-known associated or underlying diseases (e.g., all degrees of chronic obstructive pulmonary disease [COPD] (J44.0x) [17], bronchiectasis (J47), asthma (J45.1), sequela of prior pulmonary TB (B.90.9), Sjögren syndrome (M35.0), gastro-esophageal reflux disease (K21.0) will not change the DRG and accordingly not the cost weight.

In 2019, the national base rate is $€ 3544.97$ [18]. By multiplying that amount with a cost weight of 0.937 for G-DRG E76C, the mean effective reimbursement (without considering administrative surcharges such as for being a training center) for treating a non-CF NTM over 14 days is $€ 3321.64$. That amount will not change with increasing or decreasing age, gender, or the number of performed OPS. Reimbursement only increases in the rare cases when surgery due to NTM-PD is required in differential diagnosis to neoplasms of obscure origins or in case of destroyed lung lobes, due to specific surgical OPS and postoperative ventilation.

Of note, coding A.31x (NTM as a secondary diagnosis (ND) does not increase the relative weight in all selected scenarios.

To calculate the average daily reimbursement per hospital bed the reimbursement of 3321.64 has to be divided by mean length of stay for E76C. Using the respective values as described in [16] results in $(€ 3544.97 \times 0.937) / 6.4=€ 519.0$.

\section{Discussion}

To our knowledge, our study analyzes for the first time the reimbursement structure for the treatment of pulmonary NTM disease in a high-income country. In particular, we have compared the different modes used in the outpatient and inpatient setting and their economic effects. As our cost calculations demonstrate, done separately for each of the two sectors of healthcare services in Germany, guideline-based diagnosing and treatment of MAC-PD in the outpatient setting is low-paying, with the bulk of reimbursement (70.7\%) going to medication. Reimbursement for physicians' services to a MAC-PD does not exceed $11.3 \%$ of the total, which is equal to $€ 32.65$ per month. By comparison, the reimbursement for a hospital stay appears attractive; the total amount for a non-surgical NTM-induced hospital stay is "capped" by the internal algorithm of the G-DRG grouper to $€ 3321.64$, irrespective of the number and type of NTM-associated diseases the respective patient is suffering from, but covers $38.3 \%$ per cent of the whole outpatient reimbursement, and that in 14 days rather than in 30 months.

Our findings may have important implications for the outcome of NTM treatment; namely, that the discrepancy between presumably well-rewarded hospital care and the remarkably low reimbursement for long-term outpatient treatment probably hampers co-operation between physicians in the two 
sectoral "silos" and may contribute to the generally poor outcomes of the disease in Germany. Diel's publication [2] revealed an increase in mid-treatment hospital stays for German patients with NTM-PD, so that on average every NTM patient is hospitalized once a year. However, it remains unclear whether the responsibility for the rapid progression of disease that leads to hospitalization should be primarily attributed to delayed initiation of treatment with inappropriate drugs, treatment interruptions due to adverse events, or negligence in adherence on the part of the patient's. Of note, in Germany, all costs for diagnosing and treating NTM-PD—with exception of a fee of $€ 10$ per hospital day for no longer than 28 hospital days per year-are free of charge to patients. Expenses for travelling to outpatient doctors as well as to a hospital are covered by the SHI in the same vein [19], so that additional costs to be borne by the patient (out of pocket expenses) are not expected to play a significant role with respect to a preference for either inpatient or outpatient treatment. New PCR tests may be useful for the rapid diagnosis of mycobacterial infection and differentiation of MTB from NTM in microbiologically positive NTN patients, as they support immediate initiation of treatment, but their use is still limited by a lack of awareness on the part of both inpatient and outpatient doctors of NTM-PD as a probable cause of pulmonary disease.

The German outcome data suggest a clear need for integrated care, with consulting and coordination between pulmonological expert centers, i.e., specialized lung clinics and outpatient doctors and beginning with the diagnosis of NTM-PD. However, how should such integrated care that aims to establish more patient-oriented care and cross-sectoral communication look in reality? A useful example exists in the pulmonology community in Europe: a most recently published systematic review of integrated disease management showed that interventions in COPD patients from 11 industrial countries (except of Germany), involving at least two different categories of healthcare providers, reduced respiratory-related hospital admissions on the short term by $32 \%$ and on the long-term (>12 months) by $41 \%$ [20]. Unfortunately, this example has not yet been applied to the apparent need for integrated care on NTM-PD.

Another example is a Germany-specific "lighthouse project" established in 2005 according to $\S 140$ SGB V, which addresses the full spectrum of morbidities and health issues for a population: "Gesundes Kinzigtal" (Healthy Kinzigtal) [21] is named for the largely residential area it covers. The Kinzigtal region, located in the Black Forest in Southwest Germany, is home to about 70,000 people. There, a joint management company of regional physicians and the participating SHI coordinates patient care between health care providers and distributes eventual cost savings achieved between the contractual partners. The Healthy Kinzigtal approach could effectively be applied to the management of NTM disease.

The two examples, however, bring to light the main limitation of our findings: they do not provide any proof that integrated care will also result in improved outcome in pulmonary NTM disease. To gain evidence of the advantages closer alignment of the inpatient and outpatient sector may provide, future research is urgently required. An exemplary implementation and trial of optimized care for pulmonary NTM patient should be started in the near future, supported by several major SHI, allowing nationwide participation by pulmonological expert centers for NTM treatment and their referring outpatient physicians.

To achieve integrated care for NTM-PD patients, periodic case conferences between specialized hospital colleagues and outpatient doctors, coupled with voluntary 2-day hospital check-ups twice a year, could form the core of a new model. With the objectives of maintaining the quality of life of patients, re-assessing the course of disease and to preventing pulmonary exacerbations, these two days should be sufficient to complete all scheduled investigations, such as thoracic computed tomography (TCT), bronchoscopy, and sampling for microbiological investigations. Given that the amount of $€ 519$ per day - as calculated above - would provide adequate reimbursement to the hospital, the funds required for such a program would be less than the presently expected cost of punctual hospitalization for the management of pulmonary exacerbations that most NTM-PD patients experience. Given the COPD experience, where reductions of $32 \%$ to $41 \%$ in hospitalization due to exacerbations have been 
reported, the savings should be enough to provide incentives for settled physicians and finance the intersectoral exchange, inclusive of additional administrative expenses. For conducting such pilot projects, an accompanying evaluation should be established to enable a pre-post comparison of routine SHI data.

In conclusion, integrated NTM-PD care in Germany is urgently required to improve overall treatment quality and to avoid unnecessary hospitalizations due to exacerbation. An economic trade-off between the inpatient and outpatient sector is one of the prerequisites for achieving that target. To gain evidence of that that new approach, further research in terms of nationwide pilot studies is necessary.

Supplementary Materials: The following are available online at http://www.mdpi.com/1660-4601/16/20/3795/s1, File S1: Additional explanations.

Author Contributions: R.D. developed the concept, performed the computations, and wrote the original draft. P.M. reviewed and edited the manuscript. All authors discussed the results and contributed to the final manuscript.

Funding: This research received no external funding.

Conflicts of Interest: R.D. received a fee for speaking at medical congresses supported by Insmed Inc. and for participating at advisory board meetings. P.M. received a fee for participating at an expert meeting supported by Insmed Inc.

\section{References}

1. Prevots, D.R.; Marras, T.K. Epidemiology of human pulmonary infection with nontuberculous mycobacteria: A review. Clin. Chest Med. 2015, 36, 13-34. [CrossRef] [PubMed]

2. Johnson, M.M.; Odell, J.A. Nontuberculous mycobacterial pulmonary infections. J. Thorac. Dis. 2014, 6, $210-220$. [PubMed]

3. Diel, R.; Nienhaus, A.; Ringshausen, F.C.; Richter, E.; Welte, T.; Rabe, K.F.; Loddenkemper, R. Interventions against Mycobacterium avium complex pulmonary disease: A systematic review. Chest 2018, 153, 888-921. [CrossRef] [PubMed]

4. Adjemian, J.; Olivier, K.N.; Seitz, A.E.; Holland, S.M.; Prevots, D.R. Prevalence of nontuberculous mycobacterial lung disease in U.S. Medicare beneficiaries. Am. J. Respir. Crit. Care Med. 2012, 185, 881-886. [CrossRef] [PubMed]

5. $\quad$ Ringshausen, F.C.; Apel, R.M.; Bange, F.C.; de Roux, A.; Pletz, M.W.; Rademacher, J.; Suhling, H.; Wagner, D.; Welte, T. Burden and trends of hospitalisations associated with pulmonary non-tuberculous mycobacterial infections in Germany, 2005-2011. BMC Infect. Dis. 2013, 13, 231. [CrossRef] [PubMed]

6. Park, S.C.; Kang, M.J.; Han, C.H.; Lee, S.M.; Kim, C.J.; Lee, J.M.; Kang, Y.A. Prevalence, incidence, and mortality of nontuberculous mycobacterial infection in Korea: A nationwide population-based study. BMC Pulm. Med. 2019, 19, 140. [CrossRef] [PubMed]

7. Diel, R.; Jacob, J.; Lampenius, N.; Loebinger, M.; Nienhaus, A.; Rabe, K.F.; Ringshausen, F.C. Burden of non-tuberculous mycobacterial pulmonary disease in Germany. Eur. Respir. J. 2017, 49, 1602109. [CrossRef] [PubMed]

8. Marras, T.K.; Mirsaeidi, M.; Vinnard, C.; Chan, E.D.; Eagle, G.; Zhang, R.; Zhang, Q. Guidelines-based treatment associated with improved economic outcomes in nontuberculous mycobacterial lung disease. J. Med. Econ. 2019, 10, 1-8. [CrossRef] [PubMed]

9. Federal Association of Statutory Health Insurance Physicians. Physicians Fee Schedule. Available online: http://kbv.de/html/ebm.php (accessed on 17 July 2019).

10. InEK-Institut für das Entgeltsystem im Krankenhaus. Definitionshandbuch 2019; G-DRG-Version 2019; InEK: Siegburg, Germany, 2019.

11. Ambulante Spezialärztliche Versorgung (Ambulatory Specialized Care). Available online: https://www.asvservicestelle.de/Home/ASVVerzeichnis (accessed on 8 August 2019).

12. Groot, K.B.; Weinstein, M.C.; Stijnen, T.; Heijenbrok-Kal, M.H.; Hunink, M.G. Uncertainty and patient heterogeneity in medical decision models. Med. Decis. Mak. 2010, 30, 194-205. [CrossRef] [PubMed]

13. Register for Pharmaceutical Drugs in Germany (Red List $\left.{ }^{\circledR}\right)$ 2019. Available online: http://online.rote-liste.de (accessed on 3 August 2019). 
14. Hayashi, M.; Takayanagi, N.; Kanauchi, T.; Miyahara, Y.; Yanagisawa, T.; Sugita, Y. Prognostic factors of 634 HIV-negative patients with Mycobacterium avium complex lung disease. Am. J. Respir. Crit. Care Med. 2012, 185, 575-583. [CrossRef] [PubMed]

15. Griffith, D.E.; Brown-Elliott, B.A.; Langsjoen, B.; Zhang, Y.; Pan, X.; Girard, W.; Nelson, K.; Caccitolo, J.; Alvarez, J.; Shepherd, S.; et al. Clinical and molecular analysis of macrolide resistance in Mycobacterium avium complex lung disease. Am. J. Respir. Crit. Care Med. 2006, 174, 928-934. [CrossRef] [PubMed]

16. Webgrouper of the DRG-Research-Group. Available online: https://www.drg-research-group.de/index.php? option=com_webgrouper\&Itemid=112\&view=webgrouper (accessed on 17 July 2019).

17. Marras, T.K.; Campitelli, M.A.; Kwong, J.C.; Lu, H.; Brode, S.K.; Marchand-Austin, A.; Gershon, A.S.; Jamieson, F.B. Risk of nontuberculous mycobacterial pulmonary disease with obstructive lung disease. Eur. Respir. J. 2016, 48, 928-931. [CrossRef] [PubMed]

18. Allgemeine Ortskrankenkassen (AOK) Bundesbasisfallwert und Einheitlicher Basisfallwertkorridor 2019. Available online: https://www.aokgesundheitspartner.de/bund/krankenhaus/lbfw/bfw/index.html (accessed on 14 July 2019).

19. Federal Ministry of Health. Online Companion Health Insurance. Available online: https://www. bundesgesundheitsministerium.de/fahrkosten.html (accessed on 28 September 2019).

20. Kruis, A.L.; Smidt, N.; Assendelft, W.J.; Gussekloo, J.; Boland, M.R.; Rutten-van Mölken, M.; Chavannes, N.H. Cochrane corner: Is integrated disease management for patients with COPD effective? Thorax 2014, 69, 1053-1055. [CrossRef] [PubMed]

21. Schubert, I.; Siegel, A.; Graf, E.; Farin-Glattacker, E.; Ihle, P.; Köster, I.; Stelzer, D.; Mehl, C.; Schmitz, J.; Dröge, P.; et al. Study protocol for a quasi-experimental claims-based study evaluating 10-year results of the population-based integrated healthcare model 'Gesundes Kinzigtal' (Healthy Kinzigtal): The INTEGRAL study. BMJ Open 2019, 9, e025945. [CrossRef] [PubMed]

(C) 2019 by the authors. Licensee MDPI, Basel, Switzerland. This article is an open access article distributed under the terms and conditions of the Creative Commons Attribution (CC BY) license (http://creativecommons.org/licenses/by/4.0/). 